\title{
Turismo: Llevar el Derecho al día a día
}

\author{
García Abad, Lito \\ Escuela Universitaria de Turismo de A Coruña-CENP
}

\section{RESUMEN}

La asignatura de Derecho del Grado de Turismo de la Universidad de A Coruña es una materia de primer curso de carrera con seis créditos que constituye la única aproximación al ámbito jurídico civil y mercantil de todo el grado a diferencia de lo que ocurre en su entorno universitario más próximo donde 0 bien existen más asignaturas del mismo campo de conocimiento o bien su contenido se amplía hasta los 9 créditos. Esto provoca que la enorme densidad del contenido docente, que incluye una parte general civil y una parte especial contractual, hace de la asignatura una materia árida y de muy difícil seguimiento por parte del alumnado, que se ve inmerso en una dinámica frenética de un tema cada semana y media. La experiencia puesta en marcha en los últimos años mediante la reconversión del contenido docente en temas de debate en el aula a través de ejemplos prácticos del día a día del alumnado, ha permitido una mayor implicación de este y una mejora en el rendimiento de los alumnos.

PALABRAS CLAVE: Derecho, Turismo, Docencia 


\section{CITA RECOMENDADA:}

García Abad, L.(2019): Turismo: Llevar el Derecho al día a día. En De la Torre Fernández, E. (ed.) (2019). Contextos universitarios transformadores: construíndo espazos de aprendizaxe. III Xornadas de Innovación Docente. Cufie. Universidade da Coruña. A Coruña (pág. 229-238).

DOI capítulo: https://doi.org/10.17979/spudc.9788497497121.229

DOl libro: https://doi.org/10.17979/spudc.9788497497121

\section{ABSTRACT}

The subject of Law of the Degree of Tourism of the University of A Coruña is a subject of first course of career with six credits that constitutes the only approximation to the civil and commercial legal scope of the whole degree unlike what happens in its university environment nearest where there are more subjects from the same field of knowledge or their content is extended to 9 credits. This causes that the enormous density of the teaching content, which includes a general civil part and a special contractual part, makes a dry subject and difficult to be followed by the students, who see themselves involved in a frantic dynamics that makes them comprehend a unit every week and a half. The experience launched in recent years, consisting of the restructuration of the educational content in discussion topics seen in the classroom using practical examples taken from students' day-to-day issues, has allowed students to be more involved and an improvement of their performance.

KEY WORDS: Law, Tourism, Teaching 


\section{INTRODUCCIÓN}

En el primer curso del grado de Turismo de la Universidad de A Coruña (UDC) existe la asignatura de Derecho, una materia de 6 créditos, densa en contenido, que a mayores figura en el plan docente del segundo cuatrimestre del año en el que los estudiantes se adentran por primera vez en la Universidad y se sumergen en un sistema docente básicamente distinto del que provienen, es decir, cuatro años antes de que en la práctica ejerzan su actividad profesional y, por tanto, con una probabilidad alta de que se produzcan modificaciones legislativas sustanciales en algunos de los contenidos que van a estudiar, como así ha sucedido en los últimos años: viajes combinados, reglamento europeo de protección de datos, legislación de transporte y protección de consumidores, ley de contratos del sector público, ley de procedimiento administrativo, leyes de turismo, etc.

Comparativamente, y atendiendo a una mera proximidad geográfica, en el plan de estudios del mismo grado en la Universidad de Vigo figura, en idéntico periodo lectivo, Legislación turística, con 9 créditos, y en cuarto curso Derecho turístico laboral e internacional, con 6 créditos. En la Universidad de Oviedo, Derecho civil patrimonial (6 créditos), en primer curso; Derecho turístico privado (6 créditos), en segundo, y Derecho administrativo turístico (6 créditos), en tercero. Otros casos de planes de estudio del grado en universidades españolas son similares a los reseñados y, en todo caso, sustancialmente diferentes a los de la Escuela Universitaria de Turismo de A Coruña.

$\mathrm{Si}$ se atiende a las competencias específicas que presumiblemente debe adquirir un alumno de Turismo de la UDC, se observa que de forma explícita se menciona la comprensión del marco legal en que se ejerce esa actividad económica, y como resultados del aprendizaje de un alumno que ha superado la asignatura se señalan, entre otros, el conocimiento de las instituciones jurídicas turísticas, la capacidad para manejar textos legales y jurídicos, el manejo de contratos y otros documentos y la resolución de problemas básicos de la profesión. Resultados sin duda muy ambiciosos para una materia única que se imparte a un graduado en turismo y que, de ser así, dejaría en entredicho incluso a alumnos del grado de 
Derecho, que curiosamente no incluyen de forma específica asignaturas de Derecho Turístico en sus planes de estudio.

Cuando se decide afrontar, en el curso 2014-2015, un cambio sustancial en la metodología aplicada para la docencia de la asignatura, Derecho se estructuraba en torno a un temario heredado que se resumía en diez temas, cuatro generales de derecho civil y cinco puramente contractuales que iban desde los viajes combinados hasta los contratos de alojamiento, transporte 0 restauración. Un último tema se dedicaba al régimen sancionador y a la disciplina turística.

A mayores, los diez temas habrían de impartirse en la práctica real en un periodo de 14 semanas (media hábil del periodo docente), es decir, un tema teórico cada 1,4 semanas, en las que la distribución de las cuatro horas de docencia semanal se hacía de la siguiente manera: 1,5 horas de teoría, 1 hora de seminario y 90 minutos de tutorías. Estas últimas consistían en la dedicación docente de 45 minutos cada dos semanas a cada uno de los cuatro grupos en que se dividía el aula. Es decir, cada alumno recibía en total aún no tres horas de clase semanales.

\section{PROPUESTA METODOLÓGICA}

Ante esta situación, lo que primero se hizo en el curso 2015-2016 fue una reforma de la distribución horaria, no en el número de horas totales del profesor, sino en su distribución interna, ya que era inviable otra propuesta. Así, se decidió sumar o no, en función del área temática a desarrollar cada semana, la hora de seminario a las 1,5 horas teóricas y, en su caso, utilizar las tutorías como si fueran seminarios.

En cuanto al contenido docente, la única modificación se produjo en el curso siguiente, 20162017, tras alguna previa experiencia puntual que se había realizado en el curso anterior. Esta reforma no consistió en suprimir, como cabría esperar, contenido, sino que se decidió aumentar el temario a 11 lecciones, de forma que la número 11 sería una lección transversal a las 10 primeras, sin contenido teórico específico, pero que debería servir como nexo de unión 
para articular el resto del temario en aras de facilitar los resultados de aprendizaje propuestos para el alumnado.

La nueva metodología consistía, pues, en entrelazar los contenidos teóricos con ejemplos prácticos, a ser posible de la vida cotidiana del estudiante. Se buscaba ceder protagonismo al estudiante en detrimento de la clase magistral. Como dice De las Heras (2016), "parece claro que el actual alumnado universitario debería ser quien asumiese el papel principal en su formación académica, mientras que los/as enseñantes habríamos de reconsiderar desempeñar el rol protagonista de antaño y transmutarnos en la guía del aprendizaje de los discentes". Así, por ejemplo, en lugar de estudiar y explicar los requisitos del contrato, se les plantaría que explicaran un contrato de arrendamiento del piso de cualquiera de los alumnos o de un seguro (automóvil, vivienda...) para analizar sobre ese documento qué requisitos contempla la ley y cómo podría dejar de ser válido, por nulidad o por anulabilidad, tal contrato.

Y así se hizo con la inmensa mayoría de los contenidos docentes. Se trataba, en definitiva, de poner en marcha un sistema de aprendizaje basado en la resolución de problemas y en el método del caso a la manera de los sistemas experimentados en la Universidad de Chile para sus alumnos de Derecho (Baraona, Cádiz y Villanueva, 2015).

Para conseguirlo, toda la asignatura se estructuró en torno a tres grandes hitos prácticos, sujetos a evaluación:

1) un dictamen jurídico, que obligara al alumno a manejar fuentes documentales en torno a un problema de la actualidad (pisos de uso turístico, nuevas plataformas de transporte, etcétera),

2) la redacción de un recurso administrativo como forma de resolución de conflictos surgidos ante la administración, y

3) la simulación de un juicio en torno a un conflicto de transporte turístico donde los alumnos ejerzan todos los papeles posibles a excepción del de juez, que será un invitado al efecto. 
Entre uno y otro hito práctico, el discurso en el aula acompañaba el discurso teórico con supuestos de la vida del alumno que servían de debate y ejemplo: capacidad de obrar de las personas en función de la edad, derechos y deberes de los usuarios, la relación entre normativa europea y legislación española...

El principal objetivo esperado por este cambio metodológico consistía en conseguir incrementar la atención del alumno a la explicación teórica y fijar con mayor eficacia los principales contenidos.

Indudablemente, aún así el ritmo docente es infernal y exige por parte del alumno un esfuerzo semanal paralelo fuera del aula para no quedar descolgado en el ritmo del resto de los alumnos y del profesor.

Así que, periódicamente, y especialmente en las tutorías, se decidió reforzar el estudio teórico individual con simulaciones de exámenes test a través de una aplicación informática que los alumnos podían descargar en sus teléfonos móviles, Kahoot, y que posibilita una competición en el aula en aras de conocer quién obtiene el mayor número de aciertos en los test realizados. La implicación de los alumnos se consigue, a mayores, explicándoles que alguna de esas preguntas test puede formar parte del examen final.

\section{RESULTADOS}

Los resultados obtenidos aún no son significativos debido a que se ha aplicado la metodología completa solo en dos cursos académicos y al hecho de que en ambos se han obtenido resultados muy diferentes, lo que permite suponer que también pueden haber influido otras variables vinculadas a las características de los grupos.

En general, el primer curso impartido con la nueva metodología completa (contenido y distribución horaria en el 2016-2017), presentó un porcentaje de suspensos y no presentados muy superior incluso a los de años anteriores (46,7 \% en junio) y asimismo muy alejado de los obtenidos al año siguiente (27,8 \% a la misma fecha) (véase Imagen 2). 


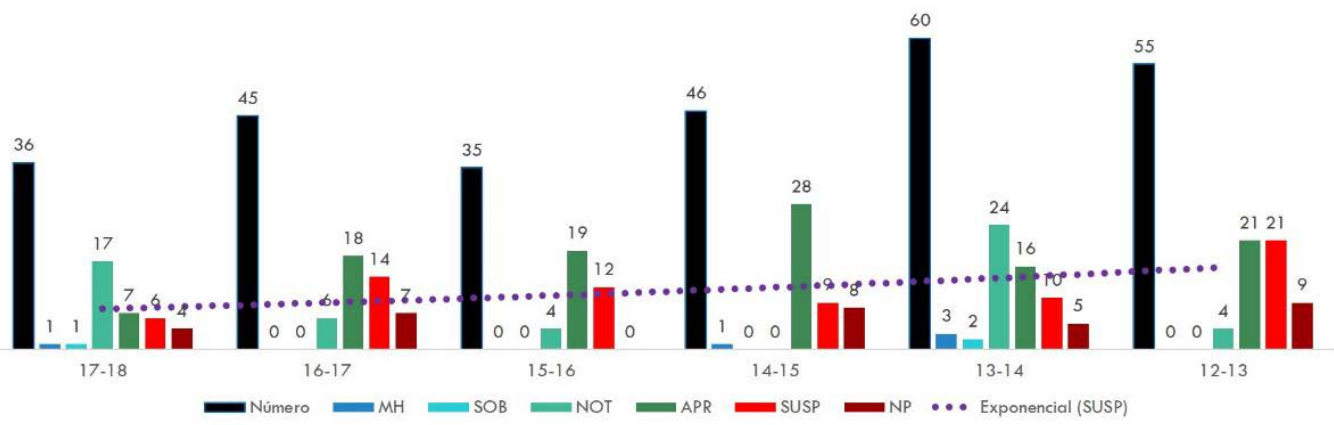

Imagen 1. Notas obtenidas en junio

Si proyectamos los resultados para un periodo más amplio, su evolución, con los datos obtenidos en la primera convocatoria (junio), muestra una ligera pendiente negativa en el número de suspensos y no presentados en el periodo 2013-2018, pero no se pueden extraer conclusiones definitivas por las distintas metodologías empleadas.

En cualquier caso, las calificaciones son un ejemplo más de que la densidad del programa docente obliga a un esfuerzo considerable en la preparación de sus contenidos, cosa que se evalúa en los exámenes en exclusividad, siendo su superación requisito inexcusable para aprobar la materia. En cambio, el compromiso del estudiante con la asignatura y su satisfacción son mayores, como así se acredita en las encuestas de satisfacción, al mismo nivel que los obtenidos por el mismo docente en otras asignaturas menos exigentes. De hecho, el mismo profesor obtiene casi idéntica puntuación en otra asignatura que requiere un menor esfuerzo comprensivo. 


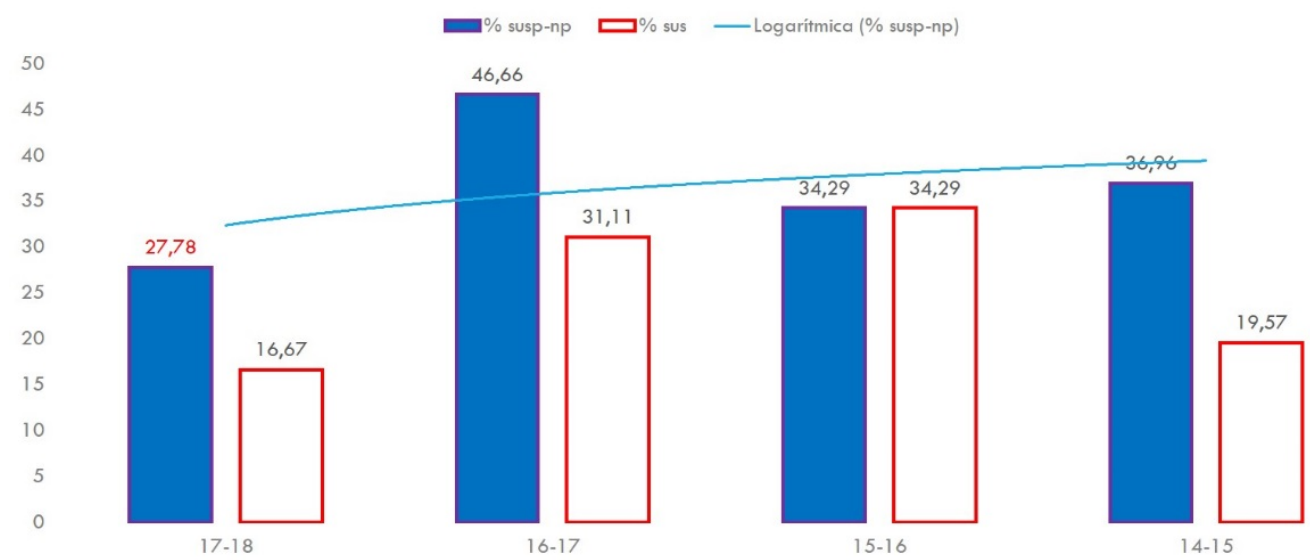

Imagen 2. Porcentajes de suspensos y no presentados (azul) y solo de suspensos (rojo)

\section{CONCLUSIONES}

Los resultados obtenidos no son en modo alguno vinculantes ni definitivos, debido al escaso periodo temporal analizado y a las enormes diferencias visualizadas en los dos cursos analizados con idéntica metodología, que apuntan a la posible influencia de otras variables inherentes a la composición de los grupos de estudiantes. Sin embargo, desde un punto de vista exclusivamente de satisfacción del alumnado, la mayor exigencia de la asignatura de Derecho no se refleja en la puntuación obtenida por el docente en las encuestas de satisfacción del alumnado, lo que parece avalar un mayor compromiso de este en el aula. La sustitución de las clases magistrales por la enseñanza basada en la resolución de problemas y por la metodología del caso han favorecido estos resultados, que habrán de confirmarse en los cursos venideros.

\section{REFERENCIAS}

Baraona, J.; Cádiz, J. \& Villanueva, 0. (2015). Thinking like a lawyer: experiencias didácticas en el aula para fortalecer la formación de un abogado. Revista Pedagogía Universitaria y Didáctica del Derecho, 2 (2), pp. 44-54. 
Casanova Ferro, G. (2004). ¿Cómo se enseña el Derecho en las Carreras de Turismo? ¿Qué se enseña? ¿Para qué se enseña? Ponencia presentada en la 16ta Conferencia Mundial de IFTTA, International Forum of Travel and Tourism Advocates, Octubre. Recuperado de https://g0o.gl/gjcJkr

De las Heras García, M. A. (2016). Cómo repensar la enseñanza del Derecho Civil para el grado en Turismo. En a Tortosa Ybáñez, M. T.; Grau Company, S., \& Álvarez Teruel, J. D. (coord.). XIV Jornadas de Redes de Investigación en Docencia Universitaria: Investigación, innovación y enseñanza universitaria: enfoques pluridisciplinares. ISBN978-84-608-79763, pp. 1131-1147.

Pérez Vallejo, A. M. (2013). Enseñanza práctica del Derecho Civil: replanteamiento metodológico y experiencias de innovación. Revista de Educación y Derecho, $\mathrm{n}^{0} 8$. Recuperado de http://revistes.ub.edu/index.php/RED/article/view/8023.

Vargas Vasserot, C. (2009). El método del caso en la enseñanza del Derecho: experiencia piloto de un piloto novel. Revista de Formación e Innovación Educativa Universitaria, 2 (4), pp. 193-206. 
